G.L. Bowman, ND, MPH

L.C. Silbert, MD, MCR

D. Howieson, PhD

H.H. Dodge, PhD

M.G. Traber, PhD

B. Frei, $\mathrm{PhD}$

J.A. Kaye, MD

J. Shannon, PhD, MPH

J.F. Quinn, MD

Correspondence \& reprint requests to Dr. Bowman:

bowmang@ohsu.edu

Editorial, page 230

Supplemental data at www.neurology.org

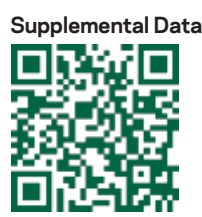

CME

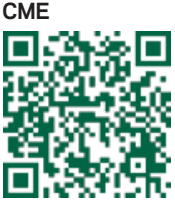

\title{
Nutrient biomarker patterns, cognitive function, and MRI measures of brain aging
}

[ㅁ]

\section{ABSTRACT}

Objective: To examine the cross-sectional relationship between nutrient status and psychometric and imaging indices of brain health in dementia-free elders.

Methods: Thirty plasma biomarkers of diet were assayed in the Oregon Brain Aging Study cohort ( $n=104)$. Principal component analysis constructed nutrient biomarker patterns (NBPs) and regression models assessed the relationship of these with cognitive and MRI outcomes.

Results: Mean age was $87 \pm 10$ years and $62 \%$ of subjects were female. Two NBPs associated with more favorable cognitive and MRI measures: one high in plasma vitamins $B(B 1, B 2, B 6$, folate, and B12), $C, D$, and $E$, and another high in plasma marine $\omega$-3 fatty acids. A third pattern characterized by high trans fat was associated with less favorable cognitive function and less total cerebral brain volume. Depression attenuated the relationship between the marine $\omega$-3 pattern and white matter hyperintensity volume.

Conclusion: Distinct nutrient biomarker patterns detected in plasma are interpretable and account for a significant degree of variance in both cognitive function and brain volume. Objective and multivariate approaches to the study of nutrition in brain health warrant further study. These findings should be confirmed in a separate population. Neurology ${ }^{\circledR}$ 2012;78:241-249

\section{GLOSSARY}

$\mathbf{A D}=$ Alzheimer disease; $\mathbf{C D R}=$ Clinical Dementia Rating; EDTA $=$ ethylenediaminetetraacetic acid; FFQ = food frequency questionnaire; $\mathrm{HDL}=$ high-density lipoprotein; HPLC = high-performance liquid chromatography; ICC = intraclass correlation coefficient; MMSE = Mini-Mental State Examination; NBP = nutrient biomarker pattern; OBAS = Oregon Brain Aging Study; PCA = principal component analysis; $\mathbf{T C B V}=$ total cerebral brain volume; $\mathbf{T I V}=$ total intracranial volume; $\mathbf{W M H}=$ white matter hyperintensity.

The epidemiology of Alzheimer disease (AD) suggests a role for nutrition. ${ }^{1-7}$ Despite studies in favor of a single or a few nutrients in the prevention of $\mathrm{AD}$, the translation to formal clinical trials testing vitamin $\mathrm{E}, \mathrm{B}$ vitamins, or docosahexaenoic acid have been disappointing. ${ }^{8-12}$ Given the interactive nature of nutrient action and metabolism, it is not surprising that a single or few nutrient approaches for neurodegenerative disease are tenuous. ${ }^{13-15}$ These results impart the rationale for novel methodologic approaches that appreciate the interactive features of nutrients and model their collective influence in the promotion of brain health.

Food frequency questionnaires (FFQ) have traditionally been used to construct dietary patterns. ${ }^{16}$ FFQ is relatively inexpensive and fairly comprehensive, but this method is subject to faulty recall of dietary intake and does not account for variability in nutrient absorption, both of which are issues in the elderly. ${ }^{17,18}$ We have recently reported a reliable blood test that assesses nutritional status in people at risk for dementia. ${ }^{19}$ In the current study, we examine the relationship of nutrient biomarkers with cognitive function and MRI.

To capture the effect of nutrients in combination, we construct nutrient biomarker patterns using principal component analysis (PCA). Cluster analysis, ${ }^{20}$ index scores,${ }^{21}$ and reduced rank

\footnotetext{
From the Departments of Neurology (G.L.B., L.C.S., D.H., H.H.D., J.A.K., J.F.Q.) and Public Health and Preventive Medicine (G.L.B., J.S.), and Center for Research in Occupational and Environmental Toxicology (J.S.), Oregon Health \& Science University, Portland; Portland VA Medical Center (J.A.K., J.S., J.F.Q.); and the Linus Pauling Institute (M.G.T., B.F.), Oregon State University, Corvallis.

References e1-e13 are available on the Neurology ${ }^{\circledR}$ Website at www.neurology.org

Study funding: Supported by NIH/NCCAM AT004777 (G.L.B.), NIH/NIA P30 AG008017 (J.A.K.), NIH/NCRR UL1 RR024140 Oregon Clinical and Translational Research Institute, and Department of Veterans Affairs, Veterans Health Administration, Office of Research and Development.

Disclosure: Author disclosures are provided at the end of the article.
} 
regression $^{22}$ have each been applied to FFQ data to assemble dietary patterns, but none have applied PCA to biological markers of diet. One goal is to define dietary patterns that promote cognitive health in the same manner that dietary approaches for hypertension have been derived and applied. ${ }^{23}$

METHODS Population. The Oregon Brain Aging Study (OBAS) was initiated in 1989 and recruited 293 communitydwelling men and women aged 65 years and older who were generally free of usual confounding factors known to modify risk for cognitive decline (i.e., vascular disease, hypertension, diabetes) to permit a purer study of aging effects on brain parameters. ${ }^{\text {e1,e2 }}$ The current cohort density is 76 and assessments are performed until death. The MMSE, neuropsychological assessments, and the Clinical Dementia Rating (CDR) reflect cognitive status. Participants attend annual study visits with a collateral historian for an evaluation by a staff neurologist, neuropsychologist, and research study member for clinical and cognitive evaluation, MRI, and blood collection. This study utilized circa 2006-2007 banked specimens and participants with a CDR $>0.5$ were excluded. Plasma, clinical, and neuropsychological data were available for 104 subjects and 42 had MRI within a month of the blood draw.

Standard protocol approval and patient consent. Informed consent was obtained from all patients for participation in this study, which was approved by the OHSU institutional review board for human study.

Nutrient biomarker acquisition and analysis. Preferably, fasting plasma was collected between 07:00 and 12:00 noon Pacific Time beginning in September 2006 and ending December 2007. Heparin plasma was deproteinized with $10 \%$ metaphosphoric acid and analyzed for ascorbic acid using high-performance liquid chromatography (HPLC) ${ }^{\mathrm{e} 3}$ Ethylenediaminetetraacetic acid (EDTA) plasma carotenoids, tocopherol, and retinol were analyzed by HPLC using diode array detector and fluorescence detection. ${ }^{\mathrm{e}}$ EDTA plasma thiamin, riboflavin, niacin, and pyridoxal 5-phoshate were analyzed by liquid chromatography-mass spectrometry/mass spectrometry. ${ }^{\text {e4 }}$ EDTA plasma folate and vitamin B12 was measured with a chemiluminescence-based assay on an Immulite analyzer (Siemens Corporation, Washington, DC). Radioimmunoassay measured EDTA plasma $25-\mathrm{OH}$ vitamin D (Immunodiagnostics Systems Inc., Scottsdale, AZ). Gas chromatography equipped with a flame ionization detector quantified plasma fatty acid concentrations. ${ }^{e 4}$ Plasma lipids were measured with standard enzymatic methods. Reliability statistics for these assays are readily available. ${ }^{19}$

Neuropsychological tests. The battery includes the following: Trail Making Test, Consortium to Establish a Registry for Alzheimer's Disease Word List acquisition and delayed recall, abbreviated Boston Naming Test, Wechsler Memory ScaleRevised Logical Memory Story A I and II and Wechsler Adult Intelligence Scale-Revised Digit Span and Block Design, and the abbreviated Geriatric Depression Scale.

Volumetric MRI acquisition and analysis. Regional volumes of interest are scaled using a 1.5-T MRI and semiautomated REGION image analysis software. Recursive regression analysis of bifeature space based on relative tissue intensities was used to separate tissue types on each coronal image. The sums of pixel areas for all slices were converted to volumetric measures by multiplying by the slice thickness for each of the following regions of interest: total white matter hyperintensity volume (WMH, includes periventricular and subcortical deep signals) and supratentorial brain volume as total cerebral brain volume (TCBV, excluding cerebellum and brainstem). Regression for brain tissue, CSF, and WMH collectively against bone creates a boundary along the inner table of the skull to determine the total intracranial volume (TIV). Additional boundaries were manually traced along the tentorium cerebelli and the superior border of the superior colliculus, the pons, and the fourth ventricle. The pituitary, vessels in the sphenoid region, and any sinuses that may have been included by the automatic regression were excluded manually. All REGION methods have an intraclass correlation coefficient (ICC) of 0.95 or better except WMH volume $(\mathrm{ICC}=0.85)$.

Covariates. PCR assay determined APOE4 carrier status. ${ }^{\text {5 }}$ Age, gender, years of education, body mass index, socioeconomic status, ${ }^{\mathrm{e} 6}$ blood pressure, current drinking and smoking, active diabetes and hypertension, active depression within the past 2 years, Hachinski scale, ${ }^{\mathrm{e} 7}$ current multivitamin use, duration of fasting, and plasma creatinine were queried from clinician interviews.

Statistical analysis. Descriptive statistics were calculated for demographic, clinical, and nutritional measures.

Nutrient biomarker pattern construction. Eight distinct nutrient biomarker patterns (NBPs) were extracted from the original set of 30 biomarkers via multivariate analysis (PCA) ${ }^{\text {e8,e9 }}$ An eigenvalue of 1.0 was set a priori to determine the NBPs to carry into hypothesis testing. Each participant receives a standardized NBP score for each pattern that corresponds to a linear combination of the plasma nutrients that load heavily within each pattern.

Cognitive $\mathrm{z}$ scores and MRI. Cognitive $z$ scores generated on the study sample $(\mathrm{n}=104)$ were conceptually combined to represent specific cognitive domains ${ }^{\mathrm{e} 10}$ and combined all together to generate a global cognitive $z$ score (table e-1 on the Neurology ${ }^{\circledR}$ Web site at www.neurology.org). MRI of the supratentorial brain divided by intracranial volume adjusted for differences in head size and the sum of the periventricular and subcortical deep white matter signals divided by the supratentorial volume adjusted for brain size prior to hypothesis testing.

Primary models. The outcomes of interest include the following: cognitive $z$ scores (global and domain specific), TCBV (\% of intracranial volume), and WMH volume (\% of TCBV). Potential confounders considered for inclusion were based on a previously recognized relationship with the outcomes and an association identified with the NBPs in this study. Two linear regression models were fit for each cognitive and MRI outcome: model 1 includes all 8 NBPs entered simultaneously plus age, gender, education, and $A P O E 4$, and model 2 is further adjusted for hypertension and depression simultaneously.

Exploratory analysis. To appreciate the proportion of variance explained in the brain outcomes by the NBPs, we examined the $R^{2}$ generated by the clinical-demographic variables initially and again with all the NBPs included in the model simultaneously. To explore the hypothesis that WMH and brain atrophy mediate the association between NBPs and cognitive function, we examined changes in significance and coefficient magnitude with and without MRI measures in the model. All analyses were performed with IBM SPSS statistical software, version 19 for Macintosh. All $p$ values reported are 2-sided. 
Table 1 Demographic and clinical characteristics ${ }^{a}$

\begin{tabular}{|c|c|}
\hline & $\mathrm{n}=104$ \\
\hline Age, y, mean (SD) & $87(10)$ \\
\hline Female, n (\%) & $64(62)$ \\
\hline Education, y, mean (SD) & $15(3)$ \\
\hline APOE4 carrier, $\mathrm{n} /$ total (\%) & 10/102 (10) \\
\hline Body mass index, mean (SD), kg/m² & $25(4)$ \\
\hline Socioeconomic status, mean (SD) & $49(10)$ \\
\hline Depression, n/total (\%) & 22/104 (21.2) \\
\hline Hypertension, n/total (\%) & $46 / 104(44.2)$ \\
\hline \multicolumn{2}{|l|}{ Blood pressure, mean (SD), mm Hg } \\
\hline Systolic & $124(17)$ \\
\hline Diastolic & $66(10)$ \\
\hline Diabetes, n/total (\%) & $5 / 104(4.8)$ \\
\hline Smoking, n/total (\%) & $2 / 104(2)$ \\
\hline Drinking, n/total (\%) & $40 / 86$ (39) \\
\hline Hachinski ischemic scale, mean (SD) & $1.7(2.0)$ \\
\hline Creatinine, plasma, mg/dL, mean (SD) & $1.0(0.3)$ \\
\hline Multivitamin use, n/total (\%) & $25 / 95(26)$ \\
\hline Fasting duration, $\mathrm{h}$, mean (SD) & $3.3(2.8)$ \\
\hline \multicolumn{2}{|l|}{ Neuropsychological tests, mean (SD) } \\
\hline Mini-Mental State Examination & $27(3)$ \\
\hline Clinical Dementia Rating & $0.17(0.24)$ \\
\hline \multicolumn{2}{|l|}{ Attention } \\
\hline Digit Span forward & $6.4(1.2)$ \\
\hline Digit Span backward & 4.7 (1.2) \\
\hline \multicolumn{2}{|l|}{ Executive function } \\
\hline Trail Making Test B & $139(79)$ \\
\hline \multicolumn{2}{|l|}{ Language } \\
\hline Boston Naming Test & $26(4)$ \\
\hline Category fluency: animals & $17(7)$ \\
\hline Category fluency: vegetables & $13(6)$ \\
\hline \multicolumn{2}{|l|}{ Memory } \\
\hline Logical Memory IA & $15(4)$ \\
\hline Logical Memory IIA & $14(5)$ \\
\hline Word-List acquisition & $19(5)$ \\
\hline Word-list recall & $5.7(2.7)$ \\
\hline \multicolumn{2}{|l|}{ Processing speed } \\
\hline Trail Making Test A & $44(23)$ \\
\hline \multicolumn{2}{|l|}{ Visual-spatial } \\
\hline Block Design & $23(8)$ \\
\hline Volumetric MRI, $\mathrm{cm}^{3}$, mean (SD) & $n=42$ \\
\hline Total intracranial volume (TIV) & $1,164(118)$ \\
\hline Total cerebral brain volume $(\mathrm{TCBV})^{\mathrm{b}}$ & $833(89)$ \\
\hline White matter hyperintensity volume (WMH) & $16(10)$ \\
\hline TCBV (\% of TIV) & $72(4)$ \\
\hline WMH (\% of TCBV) & 1.9 (1.2) \\
\hline
\end{tabular}

RESULTS Demographic, clinical, and nutritional characteristics. As expected, comorbidities and vascular risk factors were low, with the exception of hypertension (44\%). The study cohort is $62 \%$ female and $10 \%$ carry the APOE4 allele. The mean MMSE was 27 and no participants had a CDR $>0.5$ (table 1 ). The overall nutritional status in the sample population was mostly replete with prevalence of vitamin B12 deficiency $(<200 \mathrm{pg} / \mathrm{mL})$ at $7 \%$ and vitamin D deficiency $(<20 \mathrm{ng} / \mathrm{mL})$ at $25 \%$ (table e-2). Participants with MRI $(\mathrm{n}=42)$ were 85 years and older (mean age $=92.6$, SD 3.8, range 85-101). Mean age difference between those with and without MRI was 9.4 years $(p<0.0001)$. The other demographic and clinical characteristics were not different (data not shown).

Nutrient biomarker pattern construction and interpretation. Table 2 displays the composition of 8 NBPs. After the eighth NBP extraction with PCA, $72.8 \%$ of the total variance was accounted for in the original set of nutrient biomarkers (table 2, cumulative \% variance after NBP8 extraction). For the sake of reference in the following results, NBP1 is described as the BCDE pattern (all loading coefficients $>0.50$ ). NBP2 is described as the saturated fat pattern, NBP3 as the carotenoid pattern, NBP4 as the cholesterol pattern, NBP5 as the marine $\omega$ - 3 fatty acid pattern, NBP6 as the $\omega-6+$ retinol pattern, NBP7 as the lutein + high-density lipoprotein (HDL) cholesterol pattern, and NBP8 as the trans fat pattern mostly represented by trans linolelaidic acid (18:2 $\omega-6 \mathrm{t})$.

Nutrient biomarker patterns and demographic-clinical characteristics. The NBP1-BCDE and NBP5marine $\omega-3$ patterns were not associated with any demographic-clinical characteristics. The mean NBP8-trans fat score was 0.713 SD units higher in subjects with hypertension. These findings and the remaining for the other NBPs are presented in table e-3.

Nutrient biomarker patterns and cognitive function. The NBP1-BCDE and NBP8-trans fat patterns were the most significant to cognitive function (table 3). Subjects with higher BCDE scores had better global cognitive function, particularly in domains of executive, attention, and visuospatial function. Participants with higher plasma trans fat scores had worse cognitive function overall (memory, attention, language, processing speed, and global). Subjects with higher NBP5-marine $\omega$-3 scores had better executive function. Memory was better in those with higher NBP7-lutein + HDL cholesterol scores. Memory and language were worse in those with higher NBP6- $\omega-6+$ retinol scores. Adjustment for 
Table 2 Nutrient biomarker pattern construction: Pattern structure and variance explained ${ }^{\mathrm{a}}$

\begin{tabular}{|c|c|c|c|c|c|c|c|c|}
\hline Plasma nutrient biomarkers & \multicolumn{8}{|c|}{ Nutrient biomarker patterns ${ }^{b}$} \\
\hline Pyridoxal 5-phosphate (B6) & $0.847^{c}$ & & & & 0.210 & & & \\
\hline Thiamin (B1) & $0.812^{c}$ & & & & & -0.208 & & -0.269 \\
\hline Riboflavin (B2) & $0.788^{c}$ & & & & & & & 0.246 \\
\hline Folate (B9) & $0.765^{c}$ & & & & & 0.180 & & \\
\hline$\alpha$-Tocopherol (vitamin E) & $0.631^{c}$ & 0.277 & & 0.287 & & & & \\
\hline Cobalamin (B12) & $0.558^{\mathrm{c}}$ & -0.208 & 0.316 & -0.358 & & 0.279 & & \\
\hline 25-Hydroxyvitamin D & $0.555^{c}$ & -0.209 & & & 0.343 & 0.253 & & 0.215 \\
\hline$\alpha$-Linolenic acid (18:3 $\omega$-3) & & $0.866^{c}$ & & & & & & \\
\hline 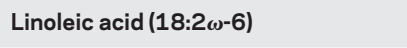 & & $0.727^{c}$ & & 0.421 & & 0.188 & & \\
\hline Trans-elaidic acid (18:1 $\omega-9 t)$ & & $0.646^{c}$ & -0.169 & -0.264 & -0.359 & & & \\
\hline$\alpha$-Carotene & & & $0.778^{\mathrm{c}}$ & & 0.271 & & 0.272 & \\
\hline$\beta$-Cryptoxanthin & & & $0.700^{c}$ & 0.165 & & & -0.164 & \\
\hline$\beta$-Carotene & 0.403 & & $0.691^{c}$ & & 0.267 & & & \\
\hline Lycopene & & & $0.552^{\mathrm{c}}$ & & -0.365 & & 0.306 & \\
\hline LDL cholesterol & & & & $0.849^{c}$ & & 0.177 & & \\
\hline Cholesterol & & 0.246 & 0.175 & $0.799^{c}$ & & 0.337 & 0.240 & \\
\hline Eicosapentaenoic acid (20:5 $\omega-3)$ & 0.180 & & 0.160 & & $0.808^{c}$ & 0.204 & 0.229 & \\
\hline Uric acid & -0.200 & 0.273 & & & -0.176 & & $-0.594^{c}$ & 0.261 \\
\hline Niacin (B3) & 0.208 & & & 0.409 & & & -0.503 & -0.402 \\
\hline Trans-linolelaidic acid (18:2 $\omega-6 t)$ & & & & 0.167 & & -0.163 & & $0.729^{c}$ \\
\hline$\%$ Variance explained by each NBP & 21 & 15 & 11 & 7 & 5.4 & 4.5 & 4.5 & 4.1 \\
\hline $\begin{array}{l}\text { Cumulative } \% \text { of variance explained } \\
\text { with each extraction }\end{array}$ & 21 & 36 & 47 & 54 & 50 & 64 & 68 & 73 \\
\hline
\end{tabular}

Abbreviations: $\mathrm{HDL}=$ high-density lipoprotein; $\mathrm{LDL}=$ low-density lipoprotein; NBP = nutrient biomarker pattern.

a Extraction method: principal component analysis. Rotation method: varimax with kaiser normalization.

${ }^{b}$ NBP interpretation is based on the strongest loading coefficients within each pattern. For example, a high NBP1 score is interpreted as high plasma vitamins $B, C, D$, and $E$. Each standardized summary score is a linear combination of the plasma nutrients that mostly represent the respective pattern. Coefficients $<0.15$ were excluded to simplify the table and emphasize dominant nutrients within each pattern.

c Considered the dominant nutrients in the pattern.

age, gender, education, $A P O E 4$, hypertension, and depression did not attenuate these relationships. Each 1-SD increase in BCDE score associated with a $0.28-S D$ increase in global cognitive score. Each $1-\mathrm{SD}$ increase in the trans fat score associated with a $0.30-S D$ decrease in global cognitive score.

Nutrient biomarker patterns and MRI. Subjects with higher plasma BCDE scores had more TCBV and those with higher trans fat scores had less TCBV. Subjects with higher marine $\omega-3$ scores had less WMH volume, but after adjustment for depression and hypertension the association was attenuated (table 4, WMH model 2). Significance of the $\omega-3$ to $\mathrm{WMH}$ was lost after adding depression to model 1 ( $p=0.030$ to 0.097 ). Adding hypertension had no effect. After stratifying by depression 
Table $3 \quad$ Nutrient biomarker patterns associated with cognitive function $(n=104)$

\begin{tabular}{|c|c|c|c|c|c|c|c|c|c|c|c|c|c|c|}
\hline & \multicolumn{2}{|l|}{ Executive } & \multicolumn{2}{|l|}{ Memory } & \multicolumn{2}{|l|}{ Attention } & \multicolumn{2}{|c|}{ Visual spatial } & \multicolumn{2}{|l|}{ Language } & \multicolumn{2}{|c|}{ Processing } & \multicolumn{2}{|l|}{ Global $^{\text {a }}$} \\
\hline \multicolumn{15}{|c|}{ NBP1 } \\
\hline SE & 0.10 & 0.10 & 0.31 & 0.31 & 0.08 & 0.08 & 0.11 & 0.11 & 0.07 & 0.07 & 0.09 & 0.09 & 0.09 & 0.09 \\
\hline \multicolumn{15}{|c|}{ NBP2 } \\
\hline SE & 0.10 & 0.10 & 0.31 & 0.31 & 0.08 & 0.08 & 0.11 & 0.11 & 0.08 & 0.08 & 0.09 & 0.09 & 0.09 & 0.09 \\
\hline \multicolumn{15}{|c|}{ NBP3 } \\
\hline$\beta$ & 0.12 & 0.02 & -0.06 & -0.07 & 0.15 & 0.15 & -0.02 & -0.03 & 0.04 & 0.03 & -0.07 & -0.06 & 0.05 & 0.05 \\
\hline SE & 0.10 & 0.10 & 0.32 & 0.32 & 0.08 & 0.08 & 0.11 & 0.11 & 0.08 & 0.08 & 0.09 & 0.09 & 0.09 & 0.09 \\
\hline \multicolumn{15}{|c|}{ NBP4 } \\
\hline \multicolumn{15}{|c|}{ NBP5 } \\
\hline$\beta$ & $-0.24^{e}$ & $-0.23^{e}$ & 0.26 & 0.24 & 0.02 & 0.02 & 0.03 & 0.01 & -0.02 & -0.04 & -0.11 & -0.11 & 0.13 & 0.12 \\
\hline SE & 0.10 & 0.10 & 0.33 & 0.33 & 0.08 & 0.08 & 0.11 & 0.11 & 0.08 & 0.08 & 0.09 & 0.09 & 0.09 & 0.09 \\
\hline \multicolumn{15}{|c|}{ NBP6 } \\
\hline$\beta$ & -0.14 & -0.12 & $-0.79^{e}$ & $-0.83^{e}$ & -0.07 & -0.06 & -0.08 & -0.10 & $-0.18^{e}$ & $-0.21^{e}$ & -0.12 & -0.11 & -0.14 & -0.15 \\
\hline SE & 0.11 & 0.11 & 0.32 & 0.33 & 0.08 & 0.08 & 0.11 & 0.11 & 0.08 & 0.08 & 0.09 & 0.09 & 0.09 & 0.09 \\
\hline \multicolumn{15}{|c|}{ NBP7 } \\
\hline$\beta$ & 0.07 & 0.05 & $0.82^{d}$ & $0.86^{d}$ & 0.01 & 0.10 & 0.03 & 0.05 & 0.06 & 0.08 & -0.05 & -0.06 & 0.13 & 0.14 \\
\hline SE & 0.09 & 0.10 & 0.29 & 0.30 & 0.07 & 0.08 & 0.10 & 0.10 & 0.07 & 0.07 & 0.08 & 0.08 & 0.08 & 0.08 \\
\hline
\end{tabular}

Abbreviations: NBP = nutrient biomarker pattern; NBP1 = BCDE; NBP2 = saturated fat; NBP3 = carotenoid; NBP4 = cholesterol; NBP5 = marine $\omega$-3; NBP6 $=\omega-6+$ retinol; NBP7 $=$ lutein + high-density lipoprotein cholesterol; NBP8 $=$ trans fat.

a Global $z$ score includes the sum of the domain $z$ scores divided by 6 . Reverse coding for executive and processing speed created a uniform direction for the coefficient (positive coefficient $=$ superior performance).

${ }^{\mathrm{b}}$ Model 1: cognitive measure $=8 \mathrm{NBPs}+$ age + gender + education + APOE4.

${ }^{c}$ Model 2: cognitive measure $=$ model $1+$ hypertension + depression.

d $p<0.01$.

e $p<0.05$.

in model 1 for $\mathrm{WMH}$ it was apparent that $\omega$-3s were significant only in those without depression $(\beta=-0.845, p=0.021)$. The unadjusted proportions of variance explained in brain volumes by each significant NBP are provided in the figure.

Exploratory analysis. Age, gender, education years, APOE 4 carrier status, depression, and hypertension together explained $46 \%$ of the variation in the global cognitive $z$ score. Adding the NBPs explained an additional $17 \%$ (global cognitive $z$ score: $R_{\text {Covariates }}^{2}=0.46 ; R_{\text {Covariates }}^{2}+\mathrm{NBPs}^{2}=$ $0.63)$. In regards to the MRI-TCBV, the covariates explained $40 \%$ of the total variation and the NBPs explained an additional 37\% (TCBV: $\left.R_{\text {Covariates }}^{2}=0.396 ; R_{\text {Covariates }+\mathrm{NBPs}^{2}}=0.766\right)$.
The covariates explained $52 \%$ of the WMH variation and the NBPs explained an additional $9 \%$ (WMH: $R_{\text {covariates }}^{2}=0.512, R_{\text {covariates }}^{2} \mathrm{NBPs}^{2}=$ $0.611)$.

To explore the hypothesis that the NBP effects on cognition are mediated through brain atrophy and WMH, we examined the changes to the association after adding MRI measures to the model. In the reduced subset with MRI available $(n=42)$, the association between NBP8-trans fat scores and global cognitive function was undetectable ( $p=0.054$ ), leaving us unable to pursue this hypothesis for trans fat. However, the relationship between NBP1$\mathrm{BCDE}$ scores and global function was maintained in this subset, and adding MRI measures did not attenuate this association $(\beta=0.614, p=0.010)$. 
Table 4 Nutrient biomarker patterns and volumetric MRI $(n=42)$

\begin{tabular}{|c|c|c|c|c|c|c|c|c|c|c|c|c|}
\hline & \multicolumn{6}{|l|}{ TCBV } & \multicolumn{6}{|l|}{ WMH } \\
\hline & \multicolumn{3}{|c|}{ Model $1^{a}$} & \multicolumn{3}{|c|}{ Model $2^{b}$} & \multicolumn{3}{|c|}{ Model $1^{a}$} & \multicolumn{3}{|c|}{ Model $2^{b}$} \\
\hline & $\boldsymbol{\beta}$ & SE & $p$ & $\boldsymbol{\beta}$ & SE & $p$ & $\boldsymbol{\beta}$ & SE & $p$ & $\boldsymbol{\beta}$ & SE & $p$ \\
\hline NBP1 BCDE & 1.29 & 0.71 & 0.08 & 1.56 & 0.60 & $0.018^{c}$ & -0.14 & 0.19 & 0.47 & -0.05 & 0.20 & 0.81 \\
\hline NBP3 carotenoid & 0.42 & 0.72 & 0.57 & 0.77 & 0.60 & 0.21 & -0.03 & 0.19 & 0.86 & 0.03 & 0.20 & 0.90 \\
\hline NBP4 cholesterol & 0.35 & 0.70 & 0.62 & 0.19 & 0.56 & 0.74 & -0.08 & 0.19 & 0.66 & -0.10 & 0.18 & 0.60 \\
\hline NBP5 marine $\omega$-3 & -0.47 & 0.73 & 0.53 & -0.75 & 0.63 & 0.25 & -0.44 & 0.21 & $0.03^{c}$ & -0.33 & 0.21 & 0.13 \\
\hline NBP6 $\omega-6$ + retinol & 0.41 & 0.81 & 0.62 & 0.12 & 0.65 & 0.86 & 0.01 & 0.21 & 0.95 & 0.02 & 0.21 & 0.91 \\
\hline
\end{tabular}

Abbreviations: $\mathrm{HDL}=$ high-density lipoprotein; TCBV = total cerebral brain volume as a \% of total intracranial volume; $\mathrm{WMH}=$ white matter hyperintensity volume as $\%$ of TCBV.

a Model 1: MRI = 8 NBP + age + gender + education + APOE4.

${ }^{\mathrm{b}}$ Model $2: \mathrm{MRI}=$ model $1+$ hypertension + depression.

${ }^{\mathrm{c}}$ Highlights statistical significance.

DISCUSSION This cross-sectional study describes the nutrient biomarker patterns identified in plasma from a sample of elders at risk for dementia. This objective and multivariate approach yielded 3 distinct NBPs significant to both cognitive function and MRI measures of brain aging. To our knowledge, this is the first study to apply principal components analysis to biological markers of diet.

Dietary patterns associated with cognitive decline or Alzheimer incidence have historically derived the patterns from FFQ data. Dietary intake can be indexed as "healthy" or "unhealthy" based on existing knowledge and examined in relation to disease risk. $^{21,24}$ Data-driven cluster analysis places subjects into exclusive dietary patterns a posterior ${ }^{20}$ and reduced rank regression combines existing knowledge and the data at hand to derive dietary patterns. ${ }^{22}$ These studies using FFQ have identified an intake higher in dark and green leafy vegetables, cruciferous vegetables, ${ }^{22}$ fish, ${ }^{25}$ and fruit ${ }^{21,22}$ and lower in organ meats, red meat, high-fat dairy, butter, ${ }^{22}$ and trans fat $^{26}$ as favorable for cognitive health. In thinking about the plasma signature of this diet, we propose that the favorable BCDE pattern and $\omega-3$ pattern would be sensitive to the frequent consumption of dark and green leafy and cruciferous vegetables, fruit, and fish. In addition, a NBP high in trans fat and retinol would be expected in people frequently consuming bakery and fried foods, margarine spreads, ${ }^{27}$ red meat, ${ }^{27}$ and offal. ${ }^{28}$ These consistencies are encouraging and provide impetus for further development of biological markers of diet.

The neuroimaging results suggest that the mechanisms through which the 2 favorable patterns (NBP1-BCDE and NBP5-marine $\omega$-3) affect cogni- tive function are distinct. Cognitive benefit gained by a plasma profile high in antioxidants $\mathrm{C}$ and $\mathrm{E}, \mathrm{B}$ vitamins, and vitamin $\mathrm{D}$ may partially operate on the neurobiology that governs rate of total brain atrophy (e.g., Alzheimer type pathology), whereas the effects of the marine $\omega$-3s may be mediated through more vascular mechanisms. ${ }^{29,30}$ The favorable relationship between the BCDE pattern and global cognitive function was maintained after adding TCBV to the model in our study. This suggests that the effects of this combination on cognition are not entirely mediated through structural changes. Other mechanisms through which this pattern may offer cognitive benefit include the promotion of hippocampal neurogenesis, ${ }^{31}$ reduction of $\beta$-secretase activity, ${ }^{32}$ oxidative stress, ${ }^{33,34}$ and hyperhomocysteinemia-induced neurotoxicity, ${ }^{35}$ and perhaps by maintaining bloodbrain barrier integrity. ${ }^{36}$

The high trans fat pattern was consistently associated with worse cognitive performance and less TCBV. Linolelaidic acid is predominantly found in bakery foods such as cookies, doughnuts, cakes, pastries, and pies. ${ }^{27}$ These foods are often prepared with hydrogenated vegetable oils to allow for a long shelf life. Higher trans fatty acid intake increases cardiovascular risk, systemic inflammation, and endothelial dysfunction, all of which may explain an association with cognition. ${ }^{37,38}$ Unfortunately, very few studies have assessed trans fat and risk for cognitive decline. $^{26}$ Trans fat may aggravate cognitive function independently and jointly through interaction with other dietary factors. ${ }^{\text {e11 }}$ Trans fat may displace DHA in neuronal membranes, but apparently does not impact the neuropathologic Alzheimer hallmarks in mice. ${ }^{39}$ The consistency of the association of plasma 
pentaenoic and docosahexaenoic acid loading together, were similar to our findings using fatty acids expressed as absolute concentrations in plasma. The

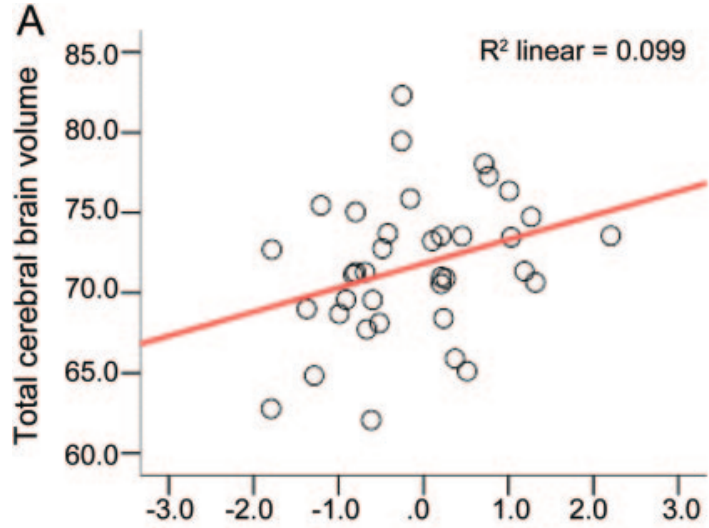

Nutrient biomarker pattern 1 score
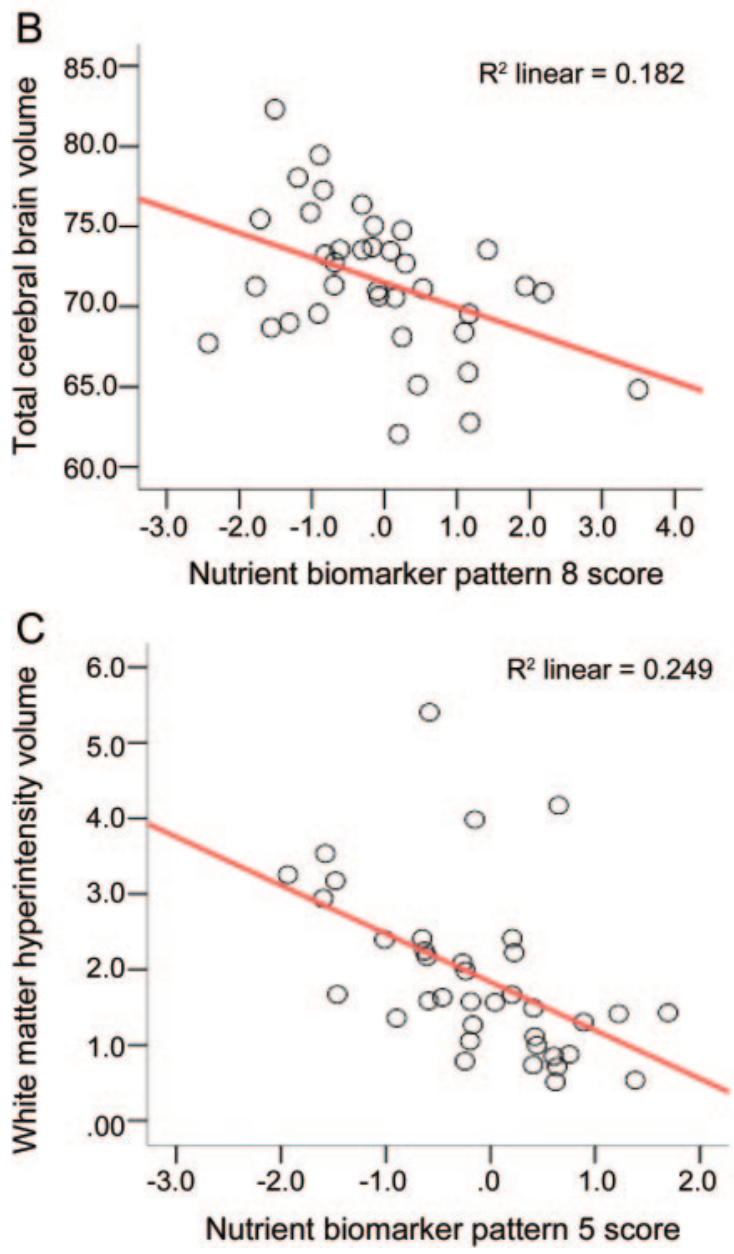

Total cerebral brain volume (A, B) expressed as a \% of total intracranial volume; white matter hyperintensity volume $(C)$ includes periventricular and subcortical deep signals expressed as a \% of total cerebral brain volume; $x$-axis represents the standardized score for NBP1-BCDE, NBP8-trans fat, and NBP5-marine $\omega$-3 patterns.

trans fat with poorer cognitive function and more brain atrophy suggests neurologic consequences in humans, but these findings need to be confirmed.

PCA of fatty acids expressed as weight percentages of total in serum and in erythrocyte membranes have been studied. ${ }^{\text {e12,e13 }}$ The patterns, including eicosainteractive metabolism of EPA and DHA, in addition to the similar dietary sources, may explain why these 2 fatty acids load together. PCA constructs the patterns on a basis of collinearity, and this "relatedness" may be partially attributed to interactive metabolism when applied to biological markers of diet. Our observation that the carotenoids (NBP3), total and low-density lipoprotein cholesterol (NBP4), saturated fats (NBP2), and the $\omega-6$ fatty acids (NBP6) load together adds further support to the notion that interactive metabolism is a contributor to NBP construction.

There are limitations of this study. PCA may require investigator decisions with the data in hand. For example, using an eigenvalue of $>1.0$ as inclusion criteria for the number of patterns extracted to carry forward into hypothesis testing may require more field-specific criteria. Our nutrient biomarkers were selected a priori capitalizing on existing knowledge of an association with neurodegeneration, but this may not reflect the ideal set. Observational studies are susceptible to residual confounding, and our cross-sectional design is not suited for inferring any causal association since the temporal relationship is unattainable. Our sample population was restricted to a relatively healthy and well-educated cohort of white, non-Hispanic elders with minimal genetic risk for $\mathrm{AD}$. These attributes may limit the generalizability of the results.

Future studies should consider validating the external consistency of these findings. The ability of NBPs to predict cognitive and brain volume changes would offer more compelling data. Gene-nutrient interactions underlying a relationship between nutrition and cognition may be important to consider since $A P O E 4$ carriers may benefit less from nutritional interventions. ${ }^{6,10,40}$ The significance of these NBPs at different stages of cognitive status are unknown. These studies will decipher the key nutrient combinations and the population best suited for intervention studies.

\section{AUTHOR CONTRIBUTIONS}

Dr. Bowman conceptualized the study, led the study procedures, the analysis, interpretation, and drafting of the manuscript. Dr. Silbert contributed to the interpretation of the neuroimaging studies and made substantive contribution to revising the manuscript for intellectual content. Dr. Howieson consulted in the interpretation of neuropsychological evaluations and made a substantive contribution in revising the manuscript for intellectual content. Dr. Dodge assisted in the data analysis for this manuscript and made a substantive contribution in revising the manuscript for intellectual content. Dr. Traber assisted in the interpretation of the data and made a substantive contribution in revising the manuscript for intellectual content. Dr. Frei assisted in the interpretation of the data and made a substantive contribution in revising the manuscript for intel- 
lectual content. Dr. Kaye made a substantive contribution in revising the manuscript for intellectual content. Dr. Shannon assisted with the conceptualization and interpretation of the results. She also made a substantive contribution in revising the manuscript for intellectual content. Dr. Quinn assisted with the conceptualization and interpretation of the results. He also made a substantive contribution in revising the manuscript for intellectual content

\section{ACKNOWLEDGMENT}

The authors thank Dara Wasserman and Robin Guariglia for data entry and management and the Oregon Brain Aging Study participants for their contributions.

\section{DISCLOSURE}

Dr. Bowman serves on the editorial board of the Journal of Alzheimer's Disease, receives salary and research support from the NIH, and insurance reimbursement for patient care. Dr. Silbert receives research support from the NIH; receives reimbursement through Medicare or commercial insurance plans for providing clinical assessment and care for patients and for intraoperative neurophysiological monitoring; and is salaried to see patients at the Portland VA Medical Center. Dr. Howieson receives salary support from the NIH/NIA and insurance reimbursement from Medicare and other sources for providing patient care. Dr. Dodge receives research support from the NIH and serves on the Scientific Review Board of the National Alzheimer's Coordinating Center. Dr. Traber receives research support from the NIH and USDA National Institute for Food and Agriculture. Dr. Frei currently serves on the Scientific Advisory Board for Unilever, Englewood Cliffs, NJ; the Almond Board Nutrition \& Health Advisory Council of the Almond Board of California, Modesto, CA; the Neutrogena Naturals Advisory Board, Los Angeles, CA; and is a consultant for Bayer Consumer Care Ltd., Basel, Switzerland. He receives research funding from NIH grants P01 AT002034 and T32 AT002688, and USANA Health Sciences, Inc., Salt Lake City, UT. Dr. Kaye receives research support from the Department of Veterans Affairs (Merit Review grant) and the NIH; directs a center that receives research support from the NIH, Elan Corporation, Intel Corporation; receives reimbursement through Medicare and commercial insurance plans for providing patient care; is salaried to see patients at the Portland VA Medical Center; serves as an unpaid Chair for the Work Group on Technology and for the National Alzheimer's Association and as an unpaid Commissioner for the Center for Aging Services and Technologies; receives an annual royalty from sales of the book, Evidence-based Dementia Practice; and serves on the editorial advisory board of Alzheimer's \& Dementia. Dr. Shannon reports no disclosures. Dr. Quinn has received honoraria for speaking from Pfizer Inc, Novartis, and Forest Laboratories, Inc. and for consulting from Phylogeny, Inc.; is a co-inventor on a patent for the use of DHA for the treatment of Alzheimer's disease; receives compensation for conducting clinical trials from Elan Corporation, Bristol-Myers Squibb, and Baxter International Inc.; and receives funding from the NIH and Department of Veterans Affairs.

Received April 10, 2011. Accepted in final form July 18, 2011.

\section{REFERENCES}

1. Morris MC, Evans DA, Bienias JL, et al. Dietary intake of antioxidant nutrients and the risk of incident Alzheimer disease in a biracial community study. JAMA 2002;287: 3230-3237.

2. Rinaldi P, Polidori MC, Metastasio A, et al. Plasma antioxidants are similarly depleted in mild cognitive impairment and in Alzheimer's disease. Neurobiol Aging 2003; 24:915-919.

3. Seshadri S, Beiser A, Selhub J, et al. Plasma homocysteine as a risk factor for dementia and Alzheimer's disease. N Engl J Med 2002;346:476-483.

4. Morris MC, Evans DA, Tangney CC, Bienias JL, Wilson RS. Fish consumption and cognitive decline with age in a large community study. Arch Neurol 2005;62:18491853.

5. Scarmeas N, Stern Y, Mayeux R, Luchsinger JA. Mediterranean diet, Alzheimer disease, and vascular mediation. Arch Neurol 2006;63:1709-1717.

6. Barberger-Gateau P, Raffaitin C, Letenneur L, et al. Dietary patterns and risk of dementia: the Three-City cohort study. Neurology 2007;69:1921-1930.

7. Schaefer EJ, Bongard V, Beiser AS, et al. Plasma phosphatidylcholine docosahexaenoic acid content and risk of dementia and Alzheimer disease: the Framingham Heart Study. Arch Neurol 2006;63:1545-1550.

8. Petersen RC, Thomas RG, Grundman M, et al. Vitamin E and donepezil for the treatment of mild cognitive impairment. N Engl J Med 2005;352:2379-2388.

9. Aisen PS, Schneider LS, Sano M, et al. High-dose B vitamin supplementation and cognitive decline in Alzheimer disease: a randomized controlled trial. JAMA 2008;300: $1774-1783$.

10. Quinn JF, Raman R, Thomas RG, et al. Docosahexaenoic acid supplementation and cognitive decline in Alzheimer disease: a randomized trial. JAMA 2010;304:1903-1911.

11. Ford AH, Flicker L, Alfonso H, et al. Vitamins B(12), $\mathrm{B}(6)$, and folic acid for cognition in older men. Neurology 2010;75:1540-1547.

12. Kang JH, Cook N, Manson J, Buring JE, Grodstein F. A randomized trial of vitamin $\mathrm{E}$ supplementation and cognitive function in women. Arch Intern Med 2006;166: 2462-2468.

13. The effect of vitamin $\mathrm{E}$ and beta carotene on the incidence of lung cancer and other cancers in male smokers: The Alpha-Tocopherol, Beta Carotene Cancer Prevention Study Group. N Engl J Med 1994;330:1029-1035.

14. Greenberg ER, Baron JA, Tosteson TD, et al. A clinical trial of antioxidant vitamins to prevent colorectal adenoma: Polyp Prevention Study Group. N Engl J Med 1994;331:141-147.

15. Hennekens CH, Buring JE, Manson JE, et al. Lack of effect of long-term supplementation with beta carotene on the incidence of malignant neoplasms and cardiovascular disease. N Engl J Med 1996;334:1145-1149.

16. Hu FB. Dietary pattern analysis: a new direction in nutritional epidemiology. Curr Opin Lipidol 2002;13:3-9.

17. Unverzagt FW, Gao S, Baiyewu O, et al. Prevalence of cognitive impairment: data from the Indianapolis Study of Health and Aging. Neurology 2001;57:1655-1662.

18. Krasinski SD, Russell RM, Samloff IM, et al. Fundic atrophic gastritis in an elderly population: effect on hemoglobin and several serum nutritional indicators. J Am Geriatr Soc 1986;34:800-806.

19. Bowman GL, Shannon J, Ho E, et al. Reliability and validity of food frequency questionnaire and nutrient biomarkers in elders with and without mild cognitive impairment. Alzheimer Dis Assoc Disord 2011;25:49-57.

20. Samieri C, Jutand MA, Feart C, Capuron L, Letenneur L, Barberger-Gateau P. Dietary patterns derived by hybrid clustering method in older people: association with cognition, mood, and self-rated health. J Am Diet Assoc 2008; 108:1461-1471.

21. Scarmeas N, Stern Y, Tang MX, Mayeux R, Luchsinger JA. Mediterranean diet and risk for Alzheimer's disease. Ann Neurol 2006;59:912-921. 
22. Gu Y, Nieves JW, Stern Y, Luchsinger JA, Scarmeas N. Food combination and Alzheimer disease risk: a protective diet. Arch Neurol 2010;67:699-706.

23. Appel LJ, Moore TJ, Obarzanek E, et al. A clinical trial of the effects of dietary patterns on blood pressure: DASH Collaborative Research Group. N Engl J Med 1997;336: 1117-1124.

24. Tangney CC, Kwasny MJ, Li H, Wilson RS, Evans DA, Morris MC. Adherence to a Mediterranean-type dietary pattern and cognitive decline in a community population. Am J Clin Nutr 2011;93:601-607.

25. Morris MC, Evans DA, Bienias JL, et al. Consumption of fish and n-3 fatty acids and risk of incident Alzheimer disease. Arch Neurol 2003;60:940-946.

26. Morris MC, Evans DA, Bienias JL, Tangney CC, Wilson RS. Dietary fat intake and 6-year cognitive change in an older biracial community population. Neurology 2004;62:1573-1579.

27. Micha R, King IB, Lemaitre RN, et al. Food sources of individual plasma phospholipid trans fatty acid isomers: the Cardiovascular Health Study. Am J Clin Nutr 2010;91:883-893.

28. Feart C, Siewe A, Samieri C, et al. Plasma retinol and association with socio-demographic and dietary characteristics of free-living older persons: the Bordeaux sample of the ThreeCity Study. Int J Vitam Nutr Res 2010;80:32-44.

29. Carmichael O, Schwarz C, Drucker D, et al. Longitudinal changes in white matter disease and cognition in the first year of the Alzheimer Disease Neuroimaging Initiative. Arch Neurol 2010;67:1370-1378.

30. Silbert LC, Nelson C, Howieson DB, Moore MM, Kaye JA. Impact of white matter hyperintensity volume progression on rate of cognitive and motor decline. Neurology 2008;71:108-113.

31. Zhao N, Zhong C, Wang Y, et al. Impaired hippocampal neurogenesis is involved in cognitive dysfunction induced by thiamine deficiency at early pre-pathological lesion stage. Neurobiol Dis 2008;29:176-185.
32. Zhang Q, Yang G, Li W, et al. Thiamine deficiency increases beta-secretase activity and accumulation of betaamyloid peptides. Neurobiol Aging 2011;32:42-53.

33. Karuppagounder SS, Xu H, Shi Q, et al. Thiamine deficiency induces oxidative stress and exacerbates the plaque pathology in Alzheimer's mouse model. Neurobiol Aging 2009;30:1587-1600.

34. Bowman GL, Dodge H, Frei B, et al. Ascorbic acid and rates of cognitive decline in Alzheimer's disease. J Alzheimers Dis 2009; 16:93-98.

35. Troen AM, Shea-Budgell M, Shukitt-Hale B, Smith DE, Selhub J, Rosenberg IH. B-vitamin deficiency causes hyperhomocysteinemia and vascular cognitive impairment in mice. Proc Natl Acad Sci USA 2008;105: 12474-12479.

36. Lehmann M, Regland B, Blennow K, Gottfries CG. Vitamin B12-B6-folate treatment improves blood-brain barrier function in patients with hyperhomocysteinaemia and mild cognitive impairment. Dement Geriatr Cogn Disord 2003;16:145-150.

37. Mozaffarian D, Katan MB, Ascherio A, Stampfer MJ, Willett WC. Trans fatty acids and cardiovascular disease. N Engl J Med 2006;354:1601-1613.

38. Lopez-Garcia E, Schulze MB, Meigs JB, et al. Consumption of trans fatty acids is related to plasma biomarkers of inflammation and endothelial dysfunction. J Nutr 2005; 135:562-566.

39. Phivilay A, Julien C, Tremblay C, et al. High dietary consumption of trans fatty acids decreases brain docosahexaenoic acid but does not alter amyloid-beta and tau pathologies in the 3xTg-AD model of Alzheimer's disease. Neuroscience 2009;159:296-307.

40. Huang TL, Zandi PP, Tucker KL, et al. Benefits of fatty fish on dementia risk are stronger for those without APOE $\epsilon 4$. Neurology 2005;65:1409-1414.

\section{It's YOUR Meeting. It's YOUR Experience.}

Cutting-edge science, education, and practice programming in YOUR area of interest.

64th AAN Annual Meeting

April 21-April 28, 2012

Ernest N. Morial Convention Center

New Orleans

Early registration ends March 28. Visit www.aan.com/golam12 today. 